\title{
Atividades extracurriculares e formação médica: diversidade e flexibilidade curricular
}

\author{
Extracurricular activities and medical formation: diversity \\ and curricular flexibility
}

\author{
lago Gonçalves Ferreira', Luciana Brandão Carreira', Nara Macedo Botelho', \\ Luís Eduardo Almeida de Souza' \\ Universidade do Estado do Pará, Belém (PA), Brasil.
}

\section{RESUMO}

As atividades extracurriculares compõem formação complementar dos acadêmicos de medicina, contribuindo com o desenvolvimento profissional e pessoal por meio da busca por novas experiências e aprendizados. O objetivo deste estudo foi realizar uma revisão narrativa sobre as atividades extracurriculares analisando características, contribuições, contradições e desafios inerentes a sua prática no contexto do ensino médico. A revisão bibliográfica ocorreu durante o período de Setembro de 2016 a Março de 2017 consultando-se as bases de dados PUBMED, SCIELO e LILACS, por se tratarem de importantes fontes abertas de literatura científica nacional, regional e mundial, bem como, incluindo-se documentos oficiais e teses acadêmicas singulares e importantes para este estudo. Os artigos apresentam a relevância destas atividades para a formação profissional em medicina, evidenciando além do benefício cognitivo, o desenvolvimento de consciência social, habilidades práticas e científicas, assim como, autonomia e proatividade. Respalda-se, na literatura, a confirmação de que as atividades complementares são indissociáveis da formação curricular oficial. Mediante a análise desta literatura, observou-se que as atividades extracurriculares influenciam variados aspectos dos acadêmicos de medicina, promovendo o desenvolvimento de diferentes habilidades, nos âmbitos técnico, social e humanístico. No entanto, quando realizadas de maneira excessiva ou em momentos inapropriados da graduação, podem prejudicar o processo de formação destes estudantes.

PALAVRAS-CHAVE: Educação médica. Estudantes de medicina. Currículo.

Recebido: Out. 22, 2016

Aceito: Mar. 20, 2017

\section{COMO CITAR ESTE ARTIGO}

Ferreira IG, Carreira LB, Botelho NM,

Souza LEA. Atividades extracurriculares

e formação médica: diversidade e

flexibilidade curricular. Interdisciplinary

Journal of Health Education. 2016

Ago-Dez;1(2):114-124. http://dx.doi

org/10.4322/ijhe.2016.022

\section{CORRESPONDÊNCIA}

Luciana Brandão Carreira

Núcleo de Pesquisa e Extensão de

Medicina, Centro de Ciências Biológicas

e da Saúde, Universidade do Estado

do Pará

Travessa Perebebuí, 2623, CEP 66087-

670, Belém (PA), Brasil

Tel.: (91) 3277-2288

lucianabrandaocarreira@gmail.com

FONTE DE FINANCIAMENTO

Recursos próprios.

\section{CONFLITO DE INTERESSE}

Os autores declararam não

haver conflitos de interesse.

O estudo foi realizado na Universidade

do Estado do Pará, Belém (PA), Brasil.

Todos os autores leram e aprovam

a versão final submetida ao

Interdisciplinary Journal of

Health Education (IJHE).

\section{ABSTRACT}

Extracurricular activities comprise complementary training of medical students, contributing to professional and personal development through new experiences and learning. The aim of this study was to perform a narrative review on extracurricular activities analyzing characteristics, contributions, contradictions and challenges inherent to their practice in the context of medical education. The bibliographic review was developed from September 2016 to March 2017, referring to the PUBMED, SCIELO and LILACS databases, due to their importance as open sources of national, regional and global scientific literature, as well as Official documents and academic thesis that are unique and important to this study. The articles present the relevance of these activities for the professional formation in medicine, evidencing besides the cognitive benefit, the development of social conscience, practical and scientific abilities, as well as autonomy and proactivity. The literature confirms that complementary activities are inseparable from official curricular training. Through the analysis of this literature, it was observed that extracurricular activities influence various aspects of medical students, promoting the development of different skills, in the technical, social and humanistic spheres. However, when carried out in an excessive manner or at inappropriate times of graduation, they may damage the training process of these students. KEYWORDS: Medical education. Medical students. Curriculum. 


\section{Introdução}

Desde o início da graduação médica, o estudante necessita adequar-se às atribuições estabelecidas pelo curso, tais como a aquisição contínua de conhecimento e a aproximação à prática profissional ${ }^{1}$. Dessa forma, na tentativa de preencher lacunas curriculares, integrar-se com colegas, suplementar carências da universidade e atender a questões profissionais, os graduandos envolvem-se em atividades extracurriculares (AEs) $)^{2}$.

A palavra "currículo" possui origem latina, tendo como significado percurso, carreira, ato de percorrer. No âmbito universitário, o currículo pode ser classificado em: currículo formal, constituído por conteúdos didáticos e atividades práticas, estabelecido oficialmente pelas universidades; e "currículo paralelo", composto pelas atividades extracurriculares que os alunos desenvolvem, subvertendo, na maioria das vezes, a estrutura curricular formal estabelecida pelas instituições ${ }^{3}$.

O termo "currículo paralelo" relaciona-se à ideia de paralelismo advinda do desempenho simultâneo dessas atividades com o currículo oficial. Nessa perspectiva, as AEs configuram-se como base complementar na formação do graduando, não imediatamente identificada, nem obrigatória, que todavia, contribuem para o desenvolvimento profissional e pessoal dos estudantes ${ }^{4}$.

Diante da complexidade e dimensão que o currículo paralelo vem adquirindo dentro dos cursos de medicina em todo o país, torna-se relevante a discussão de tal temática, a fim de promover reflexões e propostas que permitam o melhor aproveitamento dessas atividades por parte dos graduandos e do meio universitário. Sendo assim, a proposta deste artigo foi analisar as características e contribuições, bem como, dificuldades e efeitos relacionados ao desenvolvimento das atividades extracurriculares no contexto da educação médica.

\section{Metodologia}

O presente estudo trata-se de uma revisão narrativa, em que se realizou análise crítica e exploratória da literatura sobre o tema, com uma síntese qualitativa dos estudos analisados. A revisão bibliográfica ocorreu consultando-se as bases de dados PUBMED, SCIELO e LILACS, por se tratarem de importantes fontes abertas de literatura científica nacional, regional e mundial, bem como, incluindo-se documentos oficiais e teses acadêmicas singulares e importantes ao estudo. Os termos utilizados para as buscas foram: 'Educação médica', 'Estudantes de Medicina', 'Currículo' e 'Atividades Extracurriculares', em Língua Portuguesa; assim como, os termos em Inglês: 'Education, Medical'; 'Students, Medical', 'Curriculum' e 'Extracurricular Activities'. Adotou-se o período de abrangência de 2000 a 2017 para inclusão dos artigos.

A análise foi realizada durante o período de Setembro de 2016 a Março de 2017, sendo selecionados 45 documentos científicos com maior relevância e adequação à temática proposta. As informações levantadas foram sedimentadas de maneira a descrever, discutir, refletir e propor acerca das atividades extracurriculares na graduação em medicina, bem como, características, contribuições, contradições e desafios destas para a formação destes futuros profissionais.

\section{Atividades extracurriculares: contribuições e motivações}

Desde a década de 1980, acentua-se o número de estudos que evidenciam o impacto do contexto universitário, constituído pelos currículos formal e paralelo, sobre o desenvolvimento cognitivo e psicossocial do estudante na universidade ${ }^{5}$. As atividades extracurriculares apresentam aspectos diferenciados e, por esse motivo, 
as análises realizadas sobre estas oferecem aportes distintos no âmbito profissional e pessoal dos acadêmicos.

Segundo Peres et al. ${ }^{2}$, essas contribuições se concentram principalmente em cinco domínios principais: conhecimentos e habilidades acadêmicas; complexidade cognitiva; competência prática; competência interpessoal; e humanitarismo. Assim, a diferenciação do currículo por meio da participação em AEs, tem sido buscada por grande parte dos alunos, proporcionando o incremento de habilidades, conhecimento, competências e pensamento crítico, avançando para além do conteúdo formal oferecido pelas faculdades ${ }^{6-8}$.

A compreensão das causas relacionadas ao envolvimento dos graduandos nas atividades extracurriculares vem suscitando interesse em diversas investigações. De acordo com Chehuen et al. ${ }^{9}$, os alunos procuram essas iniciativas com o intuito de promover sua autoestima e realização pessoal, adquirir novos conhecimentos e experiências, bem como, respeitabilidade, reconhecimento, responsabilidade e capacidade na solução de problemas. Vieira et al. ${ }^{10}$ apontam motivações tais como o desejo em adquirir vivências que complementem o currículo, a definição profissional e, em alguns casos, razões de ordem econômica.

O currículo paralelo é desenvolvido por estudantes que ensejam a construção de uma formação médica diferenciada, concretizada pelo esforço e dedicação além do exigido na faculdade de Medicina. Por esta perspectiva, a busca por um currículo diversificado e enriquecido com estágios além da matriz curricular, apresentaria relevância na ocupação dos espaços de trabalho, em que médicos mais experientes poderiam ter melhores oportunidades em comparação aos que realizaram somente os estágios curriculares obrigatórios ${ }^{10}$.

Além deste aspecto, os concursos de Residência Médica exercem forte influência sobre a composição do currículo dos graduandos. Devido às exigências em termos de curriculum vitae e do impacto gerado pelos critérios rigorosos de avaliação, muito do que o estudante valoriza ao longo de seu curso acadêmico parece resultar dos requisitos desses concursos, ocasionando a multiplicação das atividades desempenhadas pelos alunos e o delineamento de sua formação ${ }^{11}$.

Assim, a necessidade de ingresso em um programa de Residência Médica exerce seu caráter imperativo, reforçado pela forte insegurança que cerca a passagem para a vida profissional. A Residência Médica parece ser a oportunidade e o período necessário para a obtenção de alguma segurança no exercício clínico, em um campo delimitado da atuação médica, permanecendo por mais alguns anos em trabalho supervisionado, configurando-se para alguns estudantes como um fator decisivo para uma boa formação médica ${ }^{11}$.

Os autores Roulin e Bangerter ${ }^{12}$ propõem que as motivações dos estudantes para o ingresso em AEs sejam agrupadas em duas classificações: motivações internas, como interesse e paixão sobre determinados assuntos, e motivações externas, como construção do currículo vitae e interação social. Deste modo, ao considerar-se as características das motivações dos graduandos, no que concerne ao engajamento em atividades extracurriculares, possibilita-se a estes a concepção de suas próprias vivências, não os restringindo aos núcleos comuns pré-definidos pelas universidades, estimulando a flexibilidade curricular ${ }^{13}$.

Por conseguinte, a flexibilização curricular torna-se necessária à inserção dos estudantes em cenários médicos reais, com forte componente de aprendizado profissional, observando a conciliação da vida social e do lazer à experiência de aprendizagem ${ }^{9}$. Por essa abordagem, as AEs proporcionam aos graduandos o envolvimento autônomo com temáticas e áreas que julguem interessantes e estimuladoras. 


\section{Autonomia e desenvolvimento psicossocial}

A construção de um currículo paralelo denota o envolvimento com estímulos que estão ao alcance dos estudantes, sem que tenham sido estabelecidos pelas instâncias instituídas ${ }^{2}$. À vista disso, espera-se uma postura mais diretiva destes na sua própria formação, transformando o papel do docente, antes caracterizado como transmissor de informações, para a postura de facilitador, envolvido em dinâmica mais interativa, pautada na colaboração e compreensão mútua, sendo estes elementos indispensáveis para o processo ativo de aprendizagem. De meros receptores passivos de informações previamente preparadas pelo professor, os graduandos são requisitados a construírem ativamente seu próprio conhecimento ${ }^{13}$.

Por meio da autonomia e participação ativa, os alunos podem experienciar o autêntico aprendizado com a construção e aplicação dos próprios pensamentos e ideias, o que contribui para a sua motivação com seu percurso acadêmico ${ }^{14}$. A forma de envolvimento dos graduandos nas atividades pode representar resultados diferenciados, participantes com papéis de liderança podem obter melhores desempenhos, senso de realização e autoestima, enquanto aqueles que desempenham envolvimento passivo podem apresentar desfechos mais limitados ${ }^{15,16}$.

Ademais, o desenvolvimento pessoal, social e intelectual também é beneficiado, por meio do aperfeiçoamento de habilidades, melhora das perspectivas acadêmicas e de mercado de trabalho e interação com colegas e profissioanais ${ }^{17,18}$. Alguns autores ${ }^{8,19}$ apontam que o sentimento de pertencimento a um grupo e o apoio social podem auxiliar na adaptação dos alunos à transição que a entrada na universidade representa. Ao estabelecer redes de contatos e amizades fora do ambiente de sala de aula, o estudante consolida um suporte de apoio fundamental para seu envolvimento na graduação.

Além de propiciar a interação com colegas de curso, as atividades extracurriculares também favorecem o vínculo professor-aluno, permitindo aos graduandos sentirem maior acolhimento por parte dos docentes, o que os encoraja a requisitar ajuda e sanar dúvidas. O relacionamento entre instrutores, supervisores e estudantes pode constituir-se como condição para o sucesso na adaptação e aprendizado, na medida em que, quando aluno e professor estão envolvidos em uma mesma profissão, desenvolve-se o senso de comunidade profissional, ultrapassando a barreira da idade, substituindo a relação sujeito-objeto pela colaboração mútua ${ }^{20}$.

As saúdes física e mental também podem ser beneficiadas pelo currículo paralelo, auxiliando no enfretamento de fatores estressantes da própria graduação de Medicina. Em seu tempo livre, os estudantes podem dedicar-se às atividades não relacionadas diretamente à graduação, como a prática esportiva e o aprendizado de línguas estrangeiras que também impactam diretamente sua vida futura, trazendo plenitude e satisfação pessoal ${ }^{10}$.

\section{Dificuldades, prejuízos e integração ao currículo oficial}

A realização de carga horária extraoficial pelos estudantes, por outra perspectiva, pode comprometer o tempo destinado ao descanso, lazer, atividades físicas e até mesmo ocasionar a superposição de compromissos curriculares, corroborando para uma prática acadêmica irregular ${ }^{2}$. Em muitos casos as universidades têm conhecimento do currículo paralelo desempenhado por seus estudantes, no entanto, tal ciência não se traduz na integração dessas experiências ao currículo oficial, seja por carência de recursos e/ou interesses políticos ${ }^{4}$.

Neste sentido, alguns autores destacam o impacto negativo que essas atividades podem representar para o desempenho dos estudantes, no que se refere à consciência acadêmica e às avaliações. Indaga-se ainda sobre a correlação positiva entre as AEs e 
a melhora no desempenho dos graduandos nas avaliações acadêmicas, considerando a carência de estudos que comprovem tal relação ${ }^{14,16,21,22}$.

Ressalta-se ainda o risco de estresse e problemas psicológicos associados ao sentimento de sobrecarga e à extensiva carga horária de trabalho dos alunos. Dessa forma, a maneira com que os estudantes organizam seu tempo, habilidades de estudo e compromissos pode representar maior influência em sua performance acadêmica $^{22}$.

Por esta razão, cabe as faculdades de medicina o dever de orientar seus alunos a valorização e importância do currículo formal proposto por estas, que vem sendo modificado nos últimos anos ${ }^{11}$. Ademais, devem propor ações e reformas que facilitem a conciliação de ambos os currículos, bem como, o fomento à flexibilização da matriz curricular, por meio de disciplinas eletivas, reconhecimento de horas das atividades extracurriculares e novas abordagens mais didáticas e lúdicas para o estudante.

\section{Contribuições sociais e integração a sociedade}

O processo de formação profissional não deve pressupor apenas o enriquecimento próprio e as exigências mercadológicas; ao contrário, deve permitir o diálogo entre o conhecimento acadêmico e a realidade social ${ }^{23}$. Por este entendimento, o estímulo às iniciações científicas e à docência, à formação solidária e à autonomia (iniciativas oferecidas pelas instituições) pode contribuir com uma parcela, ainda que pequena, de melhorias à sociedade ${ }^{6}$.

Tal concepção pode ser ilustrada emblematicamente, através do papel desempenhado pelas atividades extracurriculares durante a guerra da Bósnia, no início da década de 1990. Neste episódio, estudantes de medicina da Universidade de Zagreb - capital da Croácia- envolveram-se intensamente em trabalhos relacionados com os aspectos médicos da guerra. Os graduandos desenvolveram atividades como organização de cursos de ressuscitação, primeiros socorros, coleta de documentação médica de vítimas e de relatos de desrespeito aos direitos humanos, ajuda humanitária aos refugiados e atividades de comunicação ${ }^{24}$.

Segundo Gluncic et al. ${ }^{24}$, a ação dos estudantes por meio das atividades extracurriculares relacionadas à sua futura profissão e com a guerra, foi extremamente benéfica para o bem-estar psicológico e aprendizado dos jovens, bem como, para o processo de reconstrução da região, demonstrando assim, o benefício da tríade: estudantes, atividades extracurriculares e sociedade.

No âmbito nacional, destaca-se a iniciativa do Projeto Rondon. Tal projeto foi idealizado durante a década de 1970, no contexto da ditadura militar brasileira, com o objetivo de promover a intensa participação de estudantes no desenvolvimento social e econômico do país. A estratégia visava o desenvolvimento de estágios de serviço para estudantes universitários, conduzindo a participação da juventude no processo de integração nacional ${ }^{25}$.

A iniciativa contava com o apoio de universidades, por meio de diretórios e centros acadêmicos, corpos docentes e discentes. Os estudantes eram advindos preferencialmente de cursos que satisfizessem à formação de equipes multiprofissionais, no setor de saúde eram recrutados alunos de Medicina, Enfermagem, Odontologia, Farmácia, Serviço Social, dentre outros cursos superiores ${ }^{25}$.

As atividades desenvolvidas ocorriam sob diversas formas: colaboração nas consultas; aplicação de injeções, vacinas e curativos; preparo e assistência ao parto; visitas domiciliares; palestras, reuniões, cursos; aplicação de questionários para pesquisas; campanhas de saúde e primeiros socorros nas emergências ${ }^{25}$.

Dessa maneira, assim como no exemplo croata, as atividades extracurriculares propiciaram o aprimoramento de características pessoais e profissionais dos alunos envolvidos, por meio do aperfeiçoamento prático-profissional, interiorização dos 
profissionais em áreas carentes do país, preparação de mão-de-obra qualificada, integração em equipes multidisciplinares e desenvolvimento do senso cívico ${ }^{25}$.

\section{Modalidades de atividades extracurriculares}

As atividades extracurriculares buscadas pelos graduandos de medicina apresentam caráter diversificado, com formatos e objetivos variados. Originando-se por iniciativa das instituições ou dos próprios alunos, constituem em uma importante ferramenta para a flexibilidade e ampliação do conhecimento. Dentre as diversas possibilidades, destacamos algumas das mais consolidadas e discutidas na literatura Figura 1.

\section{Monitorias}

As monitorias representam a iniciação à docência, compreendendo atribuições auxiliares à atividade acadêmica regular, na qual um estudante veterano, sob a supervisão de um docente, fornece apoio ao ensino de colegas que cursam determinada disciplina pela primeira vez. Esta modalidade de AE é oferecida, geralmente, pelos departamentos das faculdades, oferecendo ou não remuneração aos alunosparticipantes ${ }^{26}$.

Além da assessoria aos professores, os graduandos podem contribuir por meio de revisões bibliográficas, auxílio à elaboração de materiais didáticos e através da prática com manequins e animais de laboratório ${ }^{6}$.

Dessa maneira, a monitoria possibilita a criação de condições para o aprofundamento teórico e desenvolvimento de habilidades dos alunos, permitindo aos monitores o esclarecimento de dúvidas e troca de experiências de maneira espontânea com um grupo de estudantes ${ }^{9,26}$.

\section{Pesquisa e Iniciação Científicas}

Segundo a Declaração sobre a Ciência e Uso do Conhecimento Científico da UNESCO, o progresso da ciência torna importante o papel das universidades para a promoção e modernização do ensino da ciência e sua coordenação em todos os níveis da educação ${ }^{27}$. Sendo assim, a pesquisa científica na graduação cumpre essa função, na medida em que, oferece a oportunidade dos estudantes engajarem-se no processo de descoberta, envolvendo-os com o meio científico, o que proporciona avaliação crítica, aplicação prática e entendimento dos conhecimentos adquiridos ${ }^{28}$.

\section{CLASSIFICAÇ̃̃O DAS ATIVIDADES EXTRACURRICULARES}

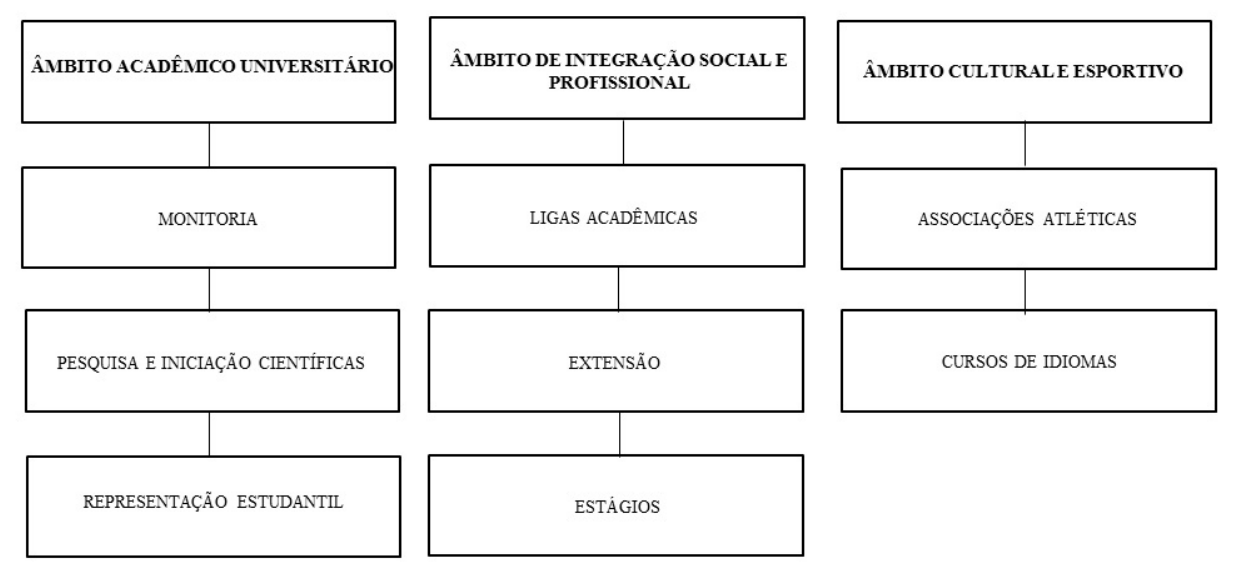

Figura 1. Classificação das atividades extracurriculares de acordo com o âmbito predominante de atuação. Fonte: Modelo proposto pelos autores. 
Por este panorama, os programas de iniciação científica (IC) têm como objetivo propiciar aos graduandos sua primeira experiência como investigador, sendo cada projeto teórico, computacional ou experimental, realizado sob a orientação de um professor ${ }^{6}$. Desse modo, promovem a inserção dos estudantes nas pesquisas científicas, pela realização de projetos e/ou estudo dirigido ${ }^{29}$.

Os programas de IC oferecem aos estudantes a possibilidade de participar em atividades como: elaboração de projetos, leitura de artigos, realização de experimentos, e até mesmo, apresentação de trabalhos em congressos e/ou publicação em periódicos científicos. Constituem-se então, como uma modalidade de atividade valorosa em termos de conhecimento, pois permitem além da aquisição de habilidades práticas, a construção de pensamento científico e estatístico ${ }^{30}$.

O incentivo financeiro propiciado por tais programas configura-se como um relevante fator para motivação dos graduandos, na medida em que permite os jovens pesquisadores prosseguir e manter a regularidade das atividades, além de oferecer sentimento de satisfação emocional e realização ${ }^{31}$.

Segundo Mileder ${ }^{32}$, o envolvimento de acadêmicos em atividades científicas, não só promoveria resultados científicos em curto prazo, mas também o potencial de melhorar a saúde em longo prazo, garantindo motivação e competência aos médico-cientistas do futuro.

Ademais, o enriquecimento do currículo com experiências como apresentações em congressos científicos e publicações pode proporcionar oportunidades nas futuras carreiras médicas dos estudantes, consistindo em aspectos relevantes a serem valorizados em seleções para programas de residência médica e/ou mestrado e doutorado. Outro aspecto importante das atividades científicas relaciona-se ao incremento do conhecimento técnico propiciado por estas, ao contribuírem para a formação de profissionais com habilidade crítica para a aplicação da Medicina Baseada em Evidências em sua prática clínica ${ }^{33}$.

Representação estudantil (Centro Acadêmico)

O Centro Acadêmico se constitui como um espaço de atuação legítima dos estudantes frente às instâncias superiores nas universidades. Essas entidades têm como propósito essencial a defesa dos interesses e direitos dos alunos, como formação profissional adequada, estrutura física apropriada e corpo docente qualificado, dentre outros aspectos necessários para a graduação com qualidade ${ }^{34}$.

Além desse papel, essas instituições possibilitam a transformação desses futuros profissionais em indivíduos com comprometimento social e visão cidadã e política acerca do contexto profissional e conjuntural no qual estão inseridos ${ }^{34}$.

\section{Ligas Acadêmicas de Medicina (LAMs)}

As Ligas Acadêmicas são organizações formadas por estudantes de Medicina de diferentes anos da graduação, tendo como objetivo o aprofundamento do estudo em determinada especialidade ou área médica. As ações dessas entidades abrangem diversos enfoques como: ações em saúde, ensino, pesquisa e extensão universitária. Tais atividades são desempenhadas sob a supervisão de docentes e profissionais vinculados a universidades ou hospitais de ensino. Dessa maneira, promovem o conhecimento e atuação dos graduandos em esferas não contempladas pelos currículos tradicionais, conduzindo seus ligantes a novas práticas de saúde, integradas com outros profissionais e com maior contato com a população $0^{35,36}$.

Ao ingressar nessas associações, os alunos participam de atividades de naturezas diversas como aulas teóricas, cursos e simpósios, além de desenvolverem projetos de pesquisa e extensão. Ademais, os graduandos tem a possbilidade de aproximarem-se da prática médica por meio da atuação em serviços médicos ou prestação de serviços à comunidade ${ }^{37}$. 
As LAMs proporcionam uma ferramenta importante para o aprendizado, que ocorre de maneira mais dinâmica, tendo em vista que sua organização é responsabilidade dos próprios alunos. Atualmente, devido ao caráter multiprofissional do atendimento em saúde, estas organizações têm aberto espaço para alunos de outros cursos como enfermagem, fisioterapia, nutrição e terapia ocupacional ${ }^{30}$.

No entanto, vale ressaltar que o interesse natural por determinada área do conhecimento e/ou especialidade não deve reduzir as perspectivas dos estudantes e limitar seu espectro de possibilidades ${ }^{38}$, do contrário, estimula-se nestes futuros profissionais o 'especialismo' precoce, e uma abordagem excessivamente cartesiana da medicina, em detrimento da perspectiva holística e generalista necessária a prática profissional.

\section{Atividades de Extensão Universitária}

Segundo o Plano Nacional de Extensão, a extensão universitária pode ser definida como um processo educativo, cultural e científico que estabelece o intercâmbio de saberes entre a sociedade e a universidade, propiciando a produção e democratização do conhecimento ${ }^{39}$. Por esta concepção, os programas e projetos de extensão atuam de forma a promover ações que gerem impacto social em cenários específicos de vulnerabilidade, aos quais determinadas comunidades e populações encontram-se inseridas ${ }^{40}$.

A abordagem social proporcionada pelas atividades de extensão contribui com a formação ético-solidária dos estudantes, uma vez que, estimula a sensibilização para questões sociais, suscitando em uma melhor qualificação e humanização da prática médica ${ }^{41}$. Dessa maneira, as instituições de ensino superior podem cumprir sua função social, atendendo ao compromisso ético de formação de seus estudantes ${ }^{6,9}$.

\section{Estágios}

Os estágios extracurriculares devido ao seu caráter eminentemente prático apresentam relevância significativa no âmbito médico-acadêmico, dado que, possibilitam aos alunos o contato precoce com a futura profissão, assim como, a vivência de aspectos relacionados ao cotidiano laboral ${ }^{13}$.

Nos estágios, exige-se dos alunos maior grau de autonomia e responsabilidade na atuação em situações da prática médica, guiando-se pelo exemplo de supervisores podem ampliar sua capacidade técnica e humanística ${ }^{42}$. Neste sentido, estas atividades representariam a chance de adquirir experiência e confiança frente aos pacientes, $\mathrm{o}$ que poderia facilitar sua futura inserção nos espaços de trabalho ${ }^{6}$.

Entretanto, vale ressaltar a necessidade de acompanhamento de tais práticas por parte das instituições, tendo em vista que, frequentemente a supervisão dos instrutores não ocorre de forma adequada, com risco de acarretar no aprendizado de conceitos e técnicas incorretos pelos estudantes ${ }^{38}$.

\section{Cursos de idiomas}

O aprendizado de línguas estrangeiras torna-se imperativo aos graduandos, tendo em vista os avanços tecnológicos e a produção científica na área médica, no sentido de favorecer à formação de profissionais atualizados e com currículo qualificado, uma vez que a maioria das publicações avançadas na área médica é proveniente de outros idiomas, principalmente a língua inglesa, e que alguns dos livros utilizados demoram cerca de três anos para serem traduzidos 9 .

Dessa maneira, ao longo da graduação, o meio acadêmico requisita do estudante o conhecimento um idioma estrangeiro, por meio da necessidade de leitura de artigos tanto para atividades curriculares como para atividades de pesquisa e publicações. Sendo assim, a busca pela realização de cursos de idiomas apresenta-se como uma das atividades extracurriculares mais relevantes. 


\section{ijhe}

Associações atléticas

As associações atléticas de medicina são organizações estudantis com o objetivo de incentivar as modalidades esportivas no meio universitário. Essas entidades não possuem fins lucrativos, sendo responsáveis pela organização de competições, treinamentos e confraternizações entre os graduandos.

As atividades esportivas ou de lazer, apresentam impacto positivo sobre o aperfeiçoamento de competências interpessoais e de liderança nos estudantes. Lazer e esporte representam uma via de emancipação com grande potencial de formação e educação humana, devendo emergir como vivências significativas para os graduandos no espaço universitário ${ }^{43,44}$.

A prática esportiva pode auxiliar o desenvolvimento da autoestima e a melhores indicadores de saúde emocional e física, além de influenciar o grau de satisfação dos estudantes com a instituição ${ }^{43}$.

\section{Papel das universidades}

As finalidades do ensino superior não são simples nem unidimensionais, ao contrário, a formação universitária pressupõe o envolvimento de um conjunto de aspectos objetivos e subjetivos, configurando-se como um desafio para as instituições a busca por projetos curriculares mais abrangentes e multidisciplinares ${ }^{6}$.

Nesse sentido, o papel da universidade diante da flexibilidade curricular propiciada pelas atividades extracurriculares pode se consolidar através do apoio e estímulo a estas iniciativas. Este incentivo pode se constituir por meio de espaços físicos, créditos curriculares, patrocínio a eventos científicos e culturais, estágios e programas de intercâmbio ${ }^{16,31,45}$.

Além destes, ressalta-se o reconhecimento acadêmico sob a forma de cerimônias de premiação, simpósios de pesquisa e divulgação em mídias sociais. Tal reconhecimento, consiste em uma importante recompensa não-financeira, proporcionando sentimento de satisfação e determinação nos alunos ${ }^{16,31,45}$.

Outro importante aspecto a ser considerado configura-se no conhecimento do contexto acadêmico por parte das instituições. Entender a realidade dos estudantes, aspirações, motivações e dificuldades no desempenho de atividades extracurriculares e analisar experiências anteriores adquiridas das organizações estudantis, consistem em ferramentas essenciais para a adequada contrapartida das universidades ${ }^{45}$.

\section{Considerações finais}

Por meio da análise da literatura, observou-se que as atividades extracurriculares têm influenciado diversos aspectos do contexto universitário dos estudantes de medicina, promovendo o desenvolvimento de habilidades, nos âmbitos técnico, social e humanístico.

Vale ressaltar a cautela que os estudantes em conjunto com as Instituições de Ensino Superior devem possuir em relação a estas atividades. Com tantas exigências nos concursos de Residência Médica, estas atividades podem perder o caráter complementar, limitando-se as perspectivas quantitativa e cumulativa registradas no curriculum Lattes. Neste sentido, as AEs podem ocasionar prejuízos para o desempenho acadêmico e a qualidade de vida dos estudantes, comprometendo sua formação por meio da redução do interesse e compromisso com as atividades do currículo formal.

Observa-se, atualmente, uma diversa gama de atividades disponíveis para os estudantes. Um valioso panorama, que facilita tanto a prática médica por meio de 
estágios supervisionados, quanto a integração acadêmica através de associações esportivas e ligas.

Em vista da multiplicidade de aspectos envolvendo as AEs, evidencia-se a relevância da discussão desta temática, com a finalidade de estimular o acompanhamento por parte das universidades do currículo paralelo desempenhado por seus estudantes. Sugere-se que outros trabalhos, delimitando o melhor modo das universidades direcionarem os alunos nessa formação, sejam desenvolvidos, assim, complementando o presente artigo e fomentando reflexões e propostas para a temática.

Destaca-se ainda que o papel das faculdades de medicina não pode se restringir à formação de médicos com conhecimento 'meramente' técnico, de outro modo, deve-se estimular o desenvolvimento de profissionais completos com conhecimento técnico-científico bem embasado e consciência social, beneficiando assim toda a sociedade. Por este aspecto, as atividades extracurriculares desempenham papel complementar à formação integral, permitindo o aprimoramento da autonomia e proatividade dos futuros médicos.

\section{Referências}

1. Rego S. Currículo paralelo em Medicina, experiência clínica e PBL: uma luz no fím do túnel? Interface Comunicacao Saude Educ. 1998;2(3):35-48. http://dx.doi.org/10.1590/S1414-32831998000200004.

2. Peres CM, Andrade AS, Garcia SB. Atividades extracurriculares: multiplicidade e diferenciação necessárias ao currículo. Rev Bras Educ Med. 2007;31(3):203-11. http://dx.doi.org/10.1590/S0100-55022007000300002.

3. Tavares CHF, Maia JA, Muniz MCH, Malta MV, Magalhães BRC, Thomaz ACP. O currículo paralelo dos estudantes da terceira série do curso médico da Universidade Federal de Alagoas. Rev Bras Educ Med. 2007;31(3):245-53. http://dx.doi. org/10.1590/S0100-55022007000300007.

4. Maia JA, Batista NA, Batista SH. O currículo no ensino superior em saúde. In: Batista AB, Batista SH, organizadores. Docência em saúde: temas e experiências. São Paulo: Senac; 2004. p. 101-134.

5. Ferreira JA, Almeida LS, Soares APC. Adaptação acadêmica em estudante do $1^{\circ}$ ano: diferenças de gênero, situação de estudante e curso. Psico, USF. 2001;6(1):1-10. http://dx.doi.org/10.1590/S1413-82712001000100002.

6. Costa BEP, Hentschke MR, Silva ACC, et al. Reflexões sobre a importância do currículo informal do estudante de medicina. Sci Med. 2012;22(3):162-8.

7. Urlings-Strop LC, Themmen AP, Stegers-Jager KM. The relationship between extracurricular activities assessed during selection and during medical school and performance. Adv Health Sci Educ Theory Pract. 2017;22(2):287-98. PMid:27812819.

8. Fares J, Saadeddin Z, Al Tabosh H, et al. Extracurricular activities associated with stress and burnout in preclinical medical students. J Epidemiol Glob Health. 2016;6(3):177-85. PMid:26644345. http://dx.doi.org/10.1016/j.jegh.2015.10.003.

9. Chehuen JÁ No, Sirimarco MT, Cândido TC, Ferreira IA, Campos RCF, Martins SC. Currículo paralelo na graduação médica na perspectiva dos estudantes. Rev Med. 2013;23(4):467-78.

10. Vieira EM, Barbieri CLA, Vilela DB, et al. O que eles fazem depois da aula? As atividades extracurriculares dos alunos de Ciências Médicas da FMRP-USP. Medicina. 2004;37(1-2):84-90.

11. Carvalho MB, Ribeiro MMF, Silva LD, Shimomura FM. A composição do Curriculum Vitae entre estudantes de medicina e seus condicionantes. Rev Bras Educ Med. 2013;37(4):483-91. http://dx.doi.org/10.1590/S0100-55022013000400003.

12. Roulin N, Bangerter A. Extracurricular activities in young applicants' resume's: what are the motives behind their involvement? Int J Psychol. 2013;48(5):871-80. PMid:22823060. http://dx.doi.org/10.1080/00207594.2012.692793.

13. Fior CA, Mercuri E. Formação universitária: o impacto das atividades não obrigatórias. In: Mercury E, Polydoro SAJ, organizadores. Estudante universitário: características e experiências de formação. Taubaté: Cabral Editora; 2003. p. 129-154.

14. Shamsudin S, Ismail SF, Al-Mamun A, Nordin SKBS. Examining the effect of extracurricular activities on academic achievements among the public university students in Malaysia. Asian Soc Sci. 2014;10(9):171-7. http://dx.doi.org/10.5539/ ass.v10n9p171.

15. Jones ML, Rush BR, Elmore RG, White BJ. Level of and motivation for extracurricular activity are associated with academic performance in the veterinary curriculum. J Vet Med Educ. 2014;41(3):275-83. PMid:24981424. http://dx.doi.org/10.3138/ jvme.1213-163R.

16. Chan YK. Investigating the relationship among extracurricular activities, learning approach and academic outcomes: A case study. Active Learn High Educ. 2016;17(3):223-33. http://dx.doi.org/10.1177/1469787416654795.

17. Lawhorn B. Extracurricular activities: the afterschool connection. OCCUP Outlook Q. 2008;52:16-21.

18. Oliveira CTD, Santos ASD, Dias ACG. Percepções de Estudantes Universitários sobre a Realização de Atividades Extracurriculares na Graduação. Psicol. Ciênc. Prof. 2016;36(4):864-76.

19. Gerrard S, Billington J. The perceived benefits of belonging to na extra curricular group within a pre-registration nursing course. Nurse Educ Pract. 2014;14(3):253-8. PMid:24295615. http://dx.doi.org/10.1016/j.nepr.2013.11.002.

20. Belikova LF. Students' attitudes toward extracurricular activity in na institution of higher learning. Russ Educ Soc. 2002;44(2):73-85. http://dx.doi.org/10.2753/RES1060-9393440273. 
21. Brint $S$, Cantwell AM. Undergraduate time use and academic outcomes: results from the University of California undergraduate experience survey 2006. Teach Coll Rec. 2010;112(9):2441-70.

22. Lumley S, Ward P, Roberts L, Mann JP. Self-reported extracurricular activity, academic success, and quality of life in UK medical students. Int J Med Educ. 2015;6:111-7. PMid:26385285. http://dx.doi.org/10.5116/ijme.55f8.5f04.

23. Lima JF. Extensão universitária como diálogo entre o saber acadêmico e a realidade social. Rev. Bras. Extensão Universitária. 2003;1(1):31-4.

24. Gluncic V, Pulanic D, Prka M, Marusic A, Marusic M. Curricular and extracurricular activitiesof medical students duringwar. Acad Med. 2001;76(1):82-7. PMid:11221772.

25. Santos MDSS, Mendes IAC. Projeto Rondon: a metodologia educativo-assistencial de trabalho dos estagiários universitários. Esc. Anna Nery. 2005;9(1):124-37.

26. Natário EG, Santos AAA. Programa de monitores para o ensino superior. Estud Psicol. 2010;27(3):355-64.

27. Organização das Nações Unidas para a Educação, a Ciência e a Cultura - UNESCO. Declaração sobre ciência e uso do conhecimento científico. Budapeste: UNESCO; 1999. p. 6-7.

28. Hu S, Kuh GD, Gayles JG. Engaging undergraduate students in research activities: Are research universities doing a better job? Innovative High Educ. 2007;32(3):167-77. http://dx.doi.org/10.1007/s10755-007-9043-y.

29. Pêgo-Fernandes PM, Mariani AW. O ensino médico além da graduação: iniciação científica. Diagn Tratamento. 2010;15:104-5.

30. Margarido MR. Atividades extracurriculares, uma opinião. Medicina. 2013;46(1):56-8.

31. Abu-Zaid A, BaHammam LO, Hijji TM, et al. Extrinsic motives to encourage extracurricular research activities: a reminder call to medical schools in Saudi Arabia. Int J Med Educ. 2017;8:99-100. PMid:28339368. http://dx.doi.org/10.5116/ijme.58bb. e1d6.

32. Mileder LP. Medical students and research: Is there a current discrepancy between education and demands? GMS Z Med Ausbild. 2014;31(2):1. PMid:24872850.

33. Nikkar-Esfahani A, Jamjoom AA, Fitzgerald JEF. Extracurricular participation in research and audit by medical students: opportunities, obstacles, motivation and outcomes. Med Teach. 2012;34(5):317-24. PMid:22471919. http://dx.doi.org/10. 3109/0142159X.2012.670324.

34. Bonfá J. Centro Acadêmico Livre de Enfermagem ontem e hoje: uma leitura da perspectiva estudantil [tese]. Vitória: Universidade Federal do Espírito Santo; 2007.

35. Oliveira CC, Souza RC, Abe EH, Silva Móz LE, Carvalho LR, Domingues MA. Undergraduate research in medical education: a descriptive study of students' views. BMC Med Educ. 2014;14(1):51. PMid:24636494. http://dx.doi. org/10.1186/1472-6920-14-51.

36. Monteiro LLF, Cunha MS, Oliveira WL, Bandeira NG, Menezes JV. Ligas Acadêmicas: o que há de positivo? Experiência da Implantação da Liga Baiana de Cirurgia Plástica. Rev. Bras. Cir. Plást. 2008;23(3):158-61.

37. Melo AP No, Martins AF, Alves PVB, Carvalho VR, Melo PGA, Pinheiro VGF. Liga Acadêmica do Pulmão: extensão universitária como parte da formação médica. Sanare. 2015;14(2):135-40.

38. Hamamoto PT Fo, Villas-Bôas PJF, Corrêa FG, et al. Normatização da abertura de ligas acadêmicas: a experiência da Faculdade de Medicina de Botucatu. Rev Bras Educ Med. 2010;34(1):160-7. http://dx.doi.org/10.1590/S0100-55022010000100019.

39. Brasil. Ministério da Educação. Plano Nacional de Extensão Universitária: Fórum de Pró-Reitores de Extensão das Universidades Públicas Brasileiras e SESu. Brasília; 2001.

40. Correa EJ, Alves MTSSB, Campos MFH, Bernardes L, Barros RMF, Crisóstimo AL. A área da saúde e a implementação da Política Nacional de Extensão. Rev. Bras. Extensão Universitária. 2006;4(2):7-13.

41. Carneiro JA, Costa FMD, Lima CC, Otaviano MR, Fróes GJ. Unimontes solidária: interação comunitária e prática médica com a extensão. Rev Bras Educ Med. 2011;35(2):283-8. http://dx.doi.org/10.1590/S0100-55022011000200019.

42. Taquette SR, Costa-Macedo LM, Alvarenga FBF. Currículo paralelo: uma realidade na formação dos estudantes de medicina da UERJ. Rev Bras Educ Med. 2003;27(3):171-6.

43. Almeida LS, Soares AP, Vasconcelos R, Capela JV, Vasconcelos JB, Corais JM, Fernandes Á. Envolvimento extracurricular e ajustamento académico: um estudo sobre as vivências dos estudantes universitários com e sem funções associativas. In: Actas do Seminário Transição para o Ensino Superior; 2000; Braga. p. 167-187.

44. Ribeiro GM, Marin EC. Universidades públicas e as políticas de esporte e lazer. Licere. 2012;15(3):1-39.

45. Freitas CCS, Figueiredo DA, Iano Y. Inclusion of extracurricular activities and student competitions in the curriculum structure for engineering education: experience based on the Brazilian reality. In: 2013 International Conference on Interactive Collaborative Learning; 2013; Kazan, Russia. p. 793-800.

\section{Contribuição dos autores}

Iago Gonçalves Ferreira: Revisão de literatura, leitura e interpretação de artigos selecionados, redação do artigo. Luciana Brandão Carreira: Orientação e supervisão acadêmica e metodológica do estudo, redação e revisão do manuscrito. Nara Macedo Botelho: Orientação e supervisão acadêmica e metodológica do estudo, redação e revisão do manuscrito. Luís Eduardo Almeida de Souza: Revisão de literatura, leitura e interpretação de artigos selecionados, redação do artigo. 